\title{
Präimplantations-Screening - Hype, Hope oder Hysterie?
}

\section{Michael von Wolff}

Prof. Dr. med., Abteilungsleiter Gynäkologische Endokrinologie und Reproduktionsmedizin, Universitäts-Frauenklinik, Inselspital Bern

Die Abstimmung zur Verfassungsänderung in der Fortpflanzungsmedizin im Juni diesen Jahres hat zu einer Diskussion geführt, die eine sachliche Analyse der Abstimmungskonsequenzen nur bedingt zulässt. Die Thematik ist so komplex, dass bei der derzeitigen Diskussion die Inhalte vielfach durchmischt werden, was wenig hilfreich ist. Deswegen soll die medizinische Relevanz insbesondere des sogenannten Präimplantations-Screenings (PIS) in der Schweiz erläutert werden.

\section{Worum geht es bei der Verfassungs- änderung?}

Die Verfassung erlaubt derzeit bei einer In-vitro-Fertilisation (IVF) nur die Entwicklung von drei und somit von nur so vielen befruchteten Eizellen wie direkt in die Gebärmutter eingesetzt werden können.

\section{Was sind die positiven Auswirkungen der Verfassungsänderung?}

Zum einen verunmöglicht die heutige Verfassung die Identifikation derjenigen befruchteten Eizellen mit dem höchsten Entwicklungspotential. Aufgrund dessen werden derzeit in der Schweiz überwiegend zwei entwickelte Eizellen transferiert, so dass inzwischen 20\% der Mehrlinge in der Schweiz auf assistierten Reproduktionstechniken beruhen [1]. Dies ist zweifelsohne eine ungute Entwicklung, da Mehrlingsschwangerschaften risikoreich sind, die Gesundheit der Kinder, aber auch die der Mütter, gefährden und damit die Fehlbildungsrate der Kinder erhöhen. Eine Verfassungsänderung würde - wie in skandinavischen Ländern - die begrüssenswerte Möglichkeit eröffnen, die Mehrlingsrate deutlich zu senken.

Zum anderen verunmöglicht die Verfassung, bei schweren Erbkrankheiten gezielt Eizellen zu kultivieren, um diejenigen zu identifizieren, die die Erbkrankheit tragen und somit nicht transferiert werden sollten. Eine Verfassungsänderung würde somit erlauben, gezielt die Erbkrankheiten in einzelnen befruchteten Eizellen zu diagnostizieren (deswegen der Begriff Präimplantations-Diagnostik, PID), die dann nicht transferiert würden. Damit könnte vermieden werden, dass eine ge-
Screening préimplantatoire -

Hype, hope ou hystérie?

Une modification de la Constitution en juin prochain permettrait la production de plus de trois ovules fécondés pour ensuite pouvoir effectuer une sélection selon des critères morphologiques, génétiques (screening préimplantatoire, SPI), et aboutir au diagnostic ciblé de maladies génétiques connues chez les deux parents (diagnostic préimplantatoire, DPI). Le SPI en particulier suscite la controverse dans les médias, mais la majorité des personnes qui en débattent semble ignorer que celui-ci n'a pas de véritable portée clinique et médicale au sens large. En effet, le petit nombre de femmes qui, pour cause de stérilité dans le couple, a recours à la fécondation in vitro (FIV), a certes la possibilité de demander un SPI, mais pour les femmes de moins de 35 ans, celui-ci ne joue pas un rôle déterminant en raison du faible nombre d'erreurs de répartition des chromosomes. Pour les femmes de plus de 35 ans en revanche, qui pourraient théoriquement tirer profit du SPI en raison d'une augmentation du nombre d'erreurs de répartition des chromosomes, il n'est en règle générale pas possible de générer suffisamment d'ovules fécondés matures pour effectuer ce SPI. Par ailleurs, les femmes qui n'ont pas de problèmes de fertilité devraient, pour pouvoir demander un SPI, effectuer une fécondation in vitro spécialement à cet effet, ce qui entraînerait un risque de malformation plus important chez l'enfant que l'atténuation du risque par un SPI. D'un point de vue médical, le SPI ne se justifie que s'il permet de réduire le nombre de fausses couches et d'accélérer les traitements FIV chez les femmes plus âgées disposant d'une réserve d'ovules élevée. 


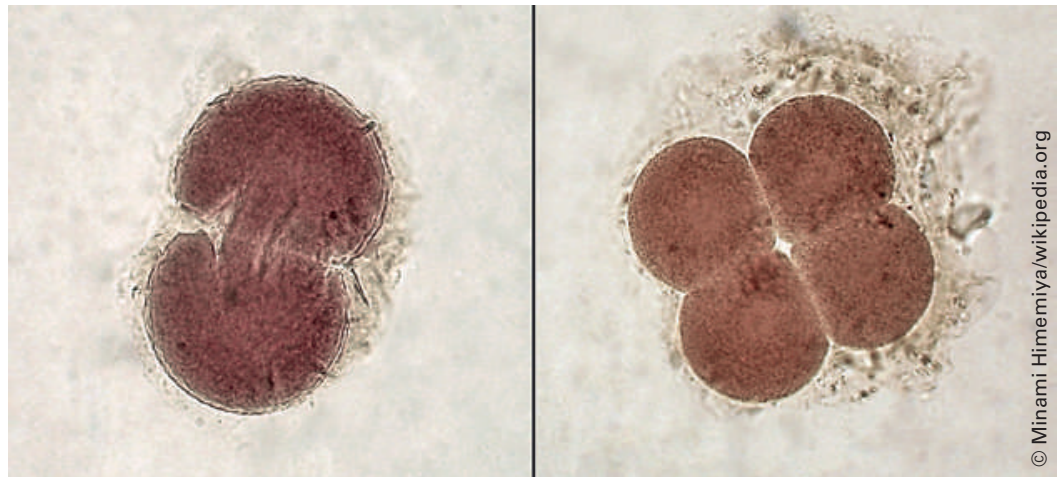

Embryonen im 2- und 4-Zellen-Stadium.

zielte Diagnostik erst in der Schwangerschaft möglich ist (Pränataldiagnostik) und daraufhin ein Schwangerschaftsabbruch gewünscht würde.

Es gibt somit zwei wesentliche Gründe für eine Verfassungsänderung, die sicherlich medizinisch nachvollziehbar, ethisch vertretbar und gesellschaftlich sinnvoll sind.

\section{Hat das PIS wirklich grosse Tragweite?}

Aber was ist mit dem Screening der entwickelten Eizellen bei einer In-vitro-Fertilisation, das sogenannte Präimplantations-Screening (PIS)? Manche Gruppierungen scheinen das PIS von der Diskussion auszuklammern, andere reduzieren die Diskussion um die Verfassungsänderung auf das PIS. Da beides nicht sinnvoll ist, sollen zum besseren Verständnis im Folgenden drei realistische Szenarien zur möglichen Anwendung des PIS dargestellt werden:

1. Frau $X$ ist jünger als 35 Jahre und macht wegen einer Sterilität eine IVF-Behandlung. Dabei könnte sie ein PIS durchführen lassen. Wohlgemerkt gehört Frau X zu einer Minderheit von Frauen in der Schweiz, da nur 2\% der Kinder nach einer IVF geboren werden [1]. Frau X müsste für das PIS zusätzlich mehrere Tausend Franken zahlen. Die Wahrscheinlichkeit einer Chromosomenaberration liegt bei Frau X unter 1\% und ist somit gering.

Frau X würde somit ein Verfahren durchführen lassen, das sehr teuer ist, eine Fehlerrate von gegenwärtig geschätzten $5 \%$ aufweist und aufgrund der altersbedingt niedrigen Wahrscheinlichkeit einer Chromosomenaberration die Fehlbildungsrate kaum senken kann. Das einzige Argument für dieses Verfahren wäre, durch die Auswahl der chromosomal intakten entwickelten Eizellen die Schwangerschaftschance bei dem ersten Transfer zu erhöhen um somit die Behandlungsdauer (bisher unbewiesen) zu verkürzen und möglicherwiese die Fehlgeburtenrate zu senken.
Deswegen: Rechtfertigen diese fraglichen Vorteile den Aufwand und die Kosten?

2. Frau Y ist älter als 35 Jahre, macht wegen einer Sterilität eine IVF-Behandlung und könnte dabei auch ein PIS durchführen lassen. Auch Frau Y gehört als IVF-Patientin zu einer Minderheit von Frauen in der Schweiz. Im Alter $>35$ Jahre ist das Risiko für eine Chromosomenanomalie in der Tat höher als mit <35 Jahren. Somit könnte in diesem Fall Frau Y theoretisch von einem PIS profitieren. Allerdings sinkt im Alter >35 Jahre die Ovarreserve, so dass nicht mehr so viele entwickelte Eizellen generiert werden können. Somit liessen sich in diesem Fall durch ein PIS zwar mit einer grösseren Wahrscheinlichkeit entwickelte Eizellen mit einer Chromosomenanomalie ausschliessen, die altersbedingt niedrige Ovarreserve schränkt die Effektivität der PIS bei Frau Y jedoch erheblich ein.

Deswegen: Rechtfertigen diese fraglichen Vorteile den Aufwand und die Kosten?

3. Frau Z ist eine gesunde 35 -jährige fertile Frau und hat einen fertilen Mann, möchte aber das Risiko minimieren, ein Kind mit einer Fehlbildung zu gebären. Da bis zu 6\% aller geborenen Kinder eine Fehlbildung aufweisen [2], ist dieser Wunsch sicherlich grundsätzlich nachvollziehbar. Frau Z könnte somit alle Frauen in der Schweiz repräsentieren.

In diesem Fall würde Frau Z, obwohl dies aufgrund der gegebenen Fertilität gar nicht erforderlich ist, eine IVF mit einem PIS durchführen lassen, um die entwickelten Eizellen mit einer Chromosomenanomalie auszuschliessen. Da das Risiko für eine Chromosomenaberration bei der Geburt bei einer 35-jährigen bei ca. 1\% liegt, könnte Frau Z das Risiko für eine Fehlbildung durch eine IVF/PIS um ca. 1\% senken. Aber: Eine IVF-Behandlung führt per se zu einer um 2\% höheren Fehlbildungsrate der Kinder [2]. Diese Erhöhung ist wahrscheinlich zum einen auf eine genetische Disposition unfruchtbarer Paare zurückzuführen, zum anderen aber auch auf die IVF-Technik als solche. Nehmen wir deswegen an, dass die IVF-Technik als solche zu einer 1\%igen Erhöhung der Fehlbildungsrate führt, würde bei dem gesunden fruchtbaren Paar, die IVF-bedingte Fehlbildungsrate um 1\% steigen. Durch das PIS kann das Paar im Gegenzug das Risiko um ca. 1\% senken. Was bleibt,

Präimplantations-Diagnostik (PID): Gezieltes Suchen nach einer bestimmten Erbkrankheit in einer befruchteten Eizelle

Präimplantations-Screening (PIS): Durchführen einer Reihe von genetischen Untersuchungen an einer befruchteten Eizelle zur Abklärung mehrere Chromosomenanomalien (Anzahl und Strukturveränderungen) 
ist bei einer IVF/PIS netto eine 0\%ige Risikominderung, da die IVF-bedingte Risikoerhöhung gleich hoch wie die Risikominderung durch das PIS ist. Deswegen: Warum sollte eine Frau, bei einer korrekt durchgeführten Aufklärung, sich dann einer solchen anstrengenden, teuren und nicht risikofreien Behandlung unterziehen, wenn diese netto gar nicht die Fehlbildungsrate verringern kann?

Fasst man den Inhalt dieser Szenarien zusammen, macht ein PIS heute und vermutlich auch in Zukunft aufgrund der biologischen und physiologischen Grenzen wie die im höherem Alter zwar zunehmende Wahr-

\section{Eine Verfassungsänderung würde erlauben, gezielt eine Erbkrankheit in einzelnen befruchteten Eizellen zu diagnostizieren.}

scheinlichkeit von Chromosomenanomalien, bei aber gleichzeitig abnehmender Ovarreserve, sowie die IVFbedingte Risikoerhöhung für Fehlbildungen kaum Sinn.

\section{Warum wurde und wird ein PIS überhaupt im Ausland durchgeführt?}

Die oben genannten Ausführungen, dass ein PIS klinisch wenig Sinn macht, stehen im Kontrast zu der relativ häufigen Nutzung des PIS, insbesondere in den USA. Wie ist dies zu erklären?

Als das PIS entwickelt wurde, nahm man an, dass die-

Ein Präimplantations-Screening (PIS) macht heute und vermutlich auch in Zukunft aufgrund biologischer und physiologischer Grenzen kaum Sinn.

ses die Erfolgschance einer IVF-Behandlung verbessern könne. Da in den USA eine IVF-Behandlung wesentlich teurer als in Europa ist, war es natürlich ein leichtes, Patientinnen von diesem vermeintlichen Vorteil zu überzeugen. Dieser Vorteil entpuppte sich jedoch als eine Fehlannahme. 2007 wurde erstmals gezeigt, dass ein PIS die Erfolgschancen einer IVF sogar reduziert [3], wahrscheinlich durch falsch positive oder falsch negative Untersuchungsergebnisse und durch den Einfluss auf den Embryo durch die Zellentnahme. Deswegen ist das PIS in europäischen Ländern, in denen die IVF-Therapien weniger teuer und die Aufklärung der Patientinnen möglicherweise besser sind, nur eine Randerscheinung.

Allerdings gibt es doch zwei Ausnahmen, bei denen ein PIS sinnvoll sein könnte:

1. Sollte eine Frau gehäuft Fehlgeburten haben, die meistens auf spontan entwickelten ChromosomenAberrationen beruhen, dann kann ein PIS helfen, das Abortrisiko zu senken.

2. Sollte eine Frau deutlich über 35 Jahre alt sein und ungewöhnlicherweise noch über eine hohe Ovarreserve verfügen, so besteht die Möglichkeit, die befruchteten Eizellen mit dem höchsten Entwicklungspotential zu identifizieren und somit die sogenannte "Time to pregnancy» zu verringern. In einem Alter, in dem die Fertilität schnell abnimmt, kann dies ein relevanter Vorteil bei der IVF-Therapie sein. Auch würden viele erfolglose Transfers mit implantationsunfähigen entwickelten Eizellen vermieden.

\section{Schlussfolgerung}

Den medizinischen Fortschritt können wir nicht aufhalten. Wir können aber versuchen, ihn zu kontrollieren. Bei dem PIS ist möglicherweise die beste Kontrolle nicht ein Verbot, sondern eine sachliche Aufklärung der Gesellschaft und der individuellen Paare. Dann kann das PIS auch kein Hype werden, gibt aber Hoffnung in medizinisch begründeten Einzelfällen. Eine Hysterie ist mit Sicherheit unangebracht.

\section{Interessenkonflikt}

Der Autor leitet die universitäre Abteilung für Gynäkologische Endokrinologie und Reproduktionsmedizin am Inselspital Bern. Weder klinisch, wissenschaftlich noch monetär bestehen im Hinblick auf die Diskussion der Verfassungsänderung Interessenkonflikte.

\section{Literatur}

1 De Geyter C, Fehr P, Moffat R, Gruber IM, von Wolff M. Twenty years' experience with the Swiss data registry for assisted reproductive medicine: outcomes, key trends and recommendations for improved practice. Swiss Med Wkly. 2015;145:w14087.

2 Davies MJ, Moore VM, Willson KJ, Van Essen P, Priest K, Scott H et al. Reproductive technologies and the risk of birth defects. N Engl J Med. 2012;366:1803-13.

3 Mastenbroek S, Twisk M, van Echten-Arends J, Sikkema-Raddatz B, Korevaar JC, Verhoeve HR et al. In vitro fertilization with preimplantation genetic screening. N Engl J Med. 2007; 357:9-17. 